\title{
Floating shoulder injuries, what parameters are important and their treatment options
}

\begin{abstract}
Introduction: Floating shoulder is the disruption of the superior shoulder suspensory complex in two or more sites. It is an uncommon injury, accounting $0.1 \%$ of all bone fractures, and has a high incidence of associated injuries, which are present in $80-90 \%$ of the cases. Currently there is no defined treatment algorithm or classification for floating shoulder. Many parameters have been described regarding the surgical criteria's, but most of them are described unevenly. Thus the instability criteria and surgical indications are unclear.
\end{abstract}

Material and methods: We made a scoping review using the methodology described by Colquhoun in 2014. We did the review of the literature of the last 10 years in Spanish and English. Then in a narrative form we described the main actual surgical criteria and options for each type of injury in the floating shoulder.

Results: To make a proper Floating Shoulder diagnosis, CT scan is recommended for a better assessment of the displacement, angulations and medialization of the injuries. At this time the decision making is based on the displacement and angulations, inferring the ligament ruptures and instability of a floating shoulder injury. Glenopolar angle of less than $30^{\circ}$ and Offset medialization of more than $20 \mathrm{~mm}$ are two parameters that can predict a bad outcome.

The displacement is an important measure in decision making. Coracoid and acromium fractures are treated surgically when displaced more than $8 \mathrm{~mm}$. Surgical Fixation of clavicle injuries is extensively recommended.

Conclusion: Floating shoulder diagnosis requires an integral assessment and complete CT workup. The treatment of floating shoulder injuries requires the assessment of each morphological injury individually. Displacement, medialization and the Glenopolar angle are the main criterias in decision making. Nowadays a floating shoulder classification or treatment protocol is needed.

Keywords: Floating Shoulder, Shoulder injury, Polytrauma, Glenoid fractures, Clavicular Fractures, Glenopolar Angle, Treatment High Enery trauma
Volume 9 Issue 4 - 2017

\author{
López-Cervantes RE,' Quintero-Hernández \\ $\mathrm{S},{ }^{2}$ Padilla-Rojas LG, ${ }^{2}$ Knierzinger $\mathrm{D},{ }^{3}$ \\ Kralinger FS, ${ }^{3}$ Gómez Acevedo JM ${ }^{4}$ \\ 'Medical Faculty, Universidad Nacional Aut \\ ${ }^{2}$ Centro Universitario de Ciencias de la Salud Universidad de \\ Guadalajara, Mexico \\ ${ }^{3}$ Trauma Department, Innsbruck University, Austria \\ ${ }^{4}$ Centro Universitario de Ciencias de la Salud Universidad de \\ Guadalajara, Mexico
}

Correspondence: Jose Maximo Gomez Acevedo, Orthopedics and Trauma Surgeon, Gabriel castanos \#26 Col. Arcos Vallarta, Zapopan, Jalisco Mexico, Tel 523000000000 ;

Email maxgomeza@hotmail.com

Received: November 08, 2017 | Published: November 24, 2017

\section{Abbreviations}

FS, Floating Shoulder; GPA, Gleno-polar Angle; SSSC, superior shoulder suspensory complex; AIS, Abbreviated Injury Scale; ATLS, Advanced Trauma Life Support $\quad$ CST, Constant Score; DASH, Disabilities of the Arm, Shoulder and Hand Score; SST, Simple Shoulder Test; TENS, Neuro-Sensitive Electro Therapy

\section{Introduction}

The floating shoulder (FS) is the disruption of the superior shoulder suspensory complex (SSSC) at two or more different points. ${ }^{1}$ The SSSC is composed by: the glenoid neck, the clavicle, the coracoid, the acromium and the $\mathrm{C} 4$ ligament complex (acromio-clavicular, coracoclavicular and coracoacromial ligaments. ${ }^{2-6}$

Scapular fractures are only $1 \%$ of all the fractures. ${ }^{2}$ Glenoid neck fractures are $18-33 \%$ of the scapular fractures and only $50 \%$ of them have a double SSSC disruption. A glenoid neck and clavicle fractures is the most common type of floating shoulder injury pattern. ${ }^{2}$ Open injuries are present in $9 \%$ of the FS cases. ${ }^{3}$

The FS is often present in polytrauma patients (AIS of more than two in two or more corporal regions). ${ }^{7}$ in such cases the FS injury can be easily overlooked. ${ }^{2}$ being belatedly diagnosed. ${ }^{1,2}$ Associated injuries are present in $80-90 \%$ of the FS cases. The most common injuries are: Head Trauma (30\%) (CET).,3,8 Thoracic trauma (30\%) and ipsilateral chest-wall injury (25\%) followed by cervical spine fractures $12 \%{ }^{2,9}$

Currently there is no classification or treatment protocol for the floating shoulder. ${ }^{1-3,8,10}$ Nowadays displacement is the main criteria of stability, ${ }^{1,2,6}$

The objective of this article is by a Scoping review of the literature, identify the main parameters which define the treatment in the floating shoulder injuries. This article has the objective to help the Trauma surgeon in the FS decision making..$^{11}$

\section{Methodology}

Thorough a working team of 6 trauma surgeons, composed by 4 surgeons specialized in shoulder surgery and treatment of the polytrauma patient, we made a narrative scoping review based on the methodology clarified by Colquhoun in $2014 .{ }^{12-14}$ The review was made following all the principles of the Helsinski declaration in its last Assemble 64a in Fortaleza, Brasil, and October 2013.

The literature review was made of the term: Floating Shoulderin the research databases: "PubMed", "Ovid", "Sciencedirect" and "Cochrane". 221 indexed publications were obtained. Inclusion criterias were publications after 2006, written in English or Spanish, 
excluding editor letters. Finally 20 publications were reviewed, adding 6 more crossed references in order to obtain the original quote. The time period was arbitrary selected because in 2006 three review articles were published, TP Goss y B D Owens. ${ }^{1}$ A Van Noort. $^{2}$ and DeFranco. ${ }^{6}$

We did a narrative review. The review responds to 4 main themes: diagnostic protocol, surgical criteria, surgical technique and postoperative and rehabilitation schemes.

\section{Discussion}

\section{Diagnostic protocol}

Most of the FS injuries are caused by High Energy trauma. ${ }^{1,3}$ In the polytrauma patient the diagnosis must follow the ATLS (Advanced Trauma Life Support) guides, searching vascular or brachial plexus injuries, gross shoulder deformity, abnormal shoulder contour, shortening of the hemi thorax, tenderness or crepitus. The patients with high energy trauma and thoracic or head trauma are the most likely to have a FS injury. ${ }^{1-3,6,15}$

Basic X-rays are a AP thorax X-ray. ${ }^{6}$ and if a scapular girdle injury is suspected at least two more X-rays projections must be taken. ${ }^{2}$ True AP view and scapula-lateral view are the most useful ones. ${ }^{1,2,6,16}$ Other useful projection is the Axillary view. ${ }^{1,6}$ having the disadvantage of being very painful. ${ }^{2}$ To asses coracoid fractures, Stryker view is recommended. ${ }^{3,6}$

The CT scan is recommended for a better assessment of the displacement, angulations and medialization of the scapular fractures. ${ }^{1-5,8,15,17,18}$

The MRI (Magnetic Resonance Imaging) use for preoperative or diagnostic proposes in the FS hasn't being reported yet. ${ }^{6}$ Jan Friederichs, says that new instability criteria's could come with the usage of this resource. ${ }^{8,15} \mathrm{~A}$ Van Noort refer that rotator cuff injuries can be diagnosed, but it's not needed for the ligament rupture diagnosis. ${ }^{2}$

\section{Surgical criteria}

Due to the low evidence based studies(Level II-IV) and lack of a standardized morphologic classification, ${ }^{1-3,8}$ the surgical criteria for the FS aren't clear, $1,2,6,17,18$

All of the open FS injuries need surgical management at least with surgical debridement in all cases. ${ }^{3,6,19}$ Izadpanah et al. recommend the clavicle fracture surgical management in all polytrauma patients to improve breathing, helping the inspiratory accessory muscles, making easier the thoracic injuries management and early mobilization. ${ }^{20}$

We divide the FS in the different type of injuries that can be present, discussing specific surgical criteria for each one of it.

\section{Glenoid neck fractures}

Glenoid neck fractures are the most common injuries in the FS. The displacement, angulations, rotation, medialization and comminution are the main surgical criteria.

In the anatomic glenoid neck fractures, surgical fixation is always advised, due to the lack of ligamentous attachments in the glenoid fragment. ${ }^{1,6,8}$

A displacement of more than $10 \mathrm{~mm}$ in Surgical Glenoid neck fractures is considered unstable and surgical management is advised. ${ }^{1,2,5,8,10}$ Edwards et al. concluded that good results can be reached with conservative treatment in glenoid neck fractures with less than $5 \mathrm{~mm}$ of displacement. ${ }^{6,21}$
The glenopolar angle (GPA) is a well known standardized measurement which normal values oscillate between $30-45^{\circ} .{ }^{10,22}$ When preserving a GPA above $30^{\circ}$ is related with greater Constant Score (CST) ${ }^{6,10,18,22}$ and Herscovici Score. ${ }^{10,20,22}$ demonstrated by some evidence III and IV studies. Romero et al demonstrated that a GPA of less than $20^{\circ}$ will produce persistent malrotation. ${ }^{4,6,23}$ Tsung-li Lin in a three group retrospective patient series reported better CST in the group with a mean GPA of $36^{\circ}$.

The glenoid offset is the distance between the clavicle medial border and the tangent line made with the humerus greater tuberosity. ${ }^{5}$ The loss of glenoid offset, is related to negative outcomes, low CST, DASH (Disabilities of the Arm, Shoulder and Hand Score) and SST (Simple Shoulder Test). ${ }^{2,5}$ DeFranco said that a difference of $30 \mathrm{~mm}$ compared to the contra lateral offset, causes: instability, bad functional outcome and pain. Dean E.P Wright said that a medialization of 10$25 \mathrm{~mm}$ will produce abduction weakness. ${ }^{4}$ Romero et al. said that a medialization of more than $25 \mathrm{~mm}$ will produce pain and abduction degree loss. ${ }^{23}$ For us an offset medialization of more than $20 \mathrm{~mm}$ can produce a bad functional outcome.

\section{Clavicle fractures}

Surgical criteria for clavicle fractures are: shortening of more than $20 \mathrm{~mm} \cdot{ }^{17,18}$ displacement of more than $10 \mathrm{~mm} \cdot{ }^{6,18}$ the absence of cortical apposition or comminution; all these indicates the need of a surgical management. ${ }^{1-6}$

Clavicular fixation is extensively recommended in the FS, because the clavicle reduction and fixation may reduce the glenoid neck fracture due to the ligamentous insertions. ${ }^{17,2,5}$

\section{Other structure injuries}

Only a few floating shoulders case reports are conformed by other shoulder girdle structure injuries. , $^{4,8,16,24,25}$

The acromio-clavicular dislocations type III or greater + another SSSC injury is considered a floating shoulder, advisable for surgical fixation. ${ }^{2}$ For the AO group, a displacement of $8 \mathrm{~mm}$ or more in coracoid or acromial fractures is indicative for surgical fixation. ${ }^{9}$ For the intra-articular glenoid fractures a displacement of more than 2 $\mathrm{mm} .{ }^{8}$ and in scapular body fractures displacement of more than 20 $\mathrm{mm}$ will require surgical fixation. ${ }^{8}$

\section{Management}

The initial management in all patients with a suspected floating shoulder injury, should be immobilization in a sling. ${ }^{25}$ The treatment for associated injuries needs to be done according to ATLS protocol.

\section{Surgical treatment}

Surgical fixation decision making should be made individually for each injury of the SSSC. There are two ways to make the surgical fixation as B D Owens and T P Goss referred. ${ }^{1}$ First to fix the clavicle in a beach chair position and then reposition the patient to dorsal decubitus for scapular fixation. The other way is by fixing both injuries in lateral decubitus. This decision has to be made according to surgeon preferences and associated injuries. ${ }^{16}$

The most preferred surgical approach for the scapular neck fracture fixation is the posterior deltoid approach. . $^{1,2,9,26}$ Also Judet and Modified Judet approaches have been described for complex fractures. ${ }^{5,16}$

Angle stable implants are being used in most of the fracture cases, being easier to place and to achieve a stable fixation. ${ }^{5,16,17}$ Non locking implants can still be used; being the reconstruction $3.5 \mathrm{~mm}$ plates the preferred ones. ${ }^{1,18}$ 
Other implants like TENS (Titanium Elastic Nails), have been used. Izadpanah et al in a case-control series with a 36 months of follow up demonstrate that in the AO type B and $\mathrm{C}$ clavicle fractures, the TENS group suffered clavicular shortening of $5 \mathrm{~mm}$ in contrast with the LCP plate group where shortening wasn't seen. ${ }^{27}$

In cases of severe comminution, threaded $\mathrm{k}$-wires or screws can be use to fix the glenoid fragment to the acromion or distal clavicle. $1,2,5,16,18$

In the Floating shoulder, fixation of other components of the SSSC, have been poorly described in limited case reports. ${ }^{4,8,16,24,25}$

\section{Conservative treatment}

The conservative treatment of the selected FS cases should be made through immobilization in a sling during the first 3-4 weeks. ${ }^{5,6,8,10}$ Free hand and elbow movement is recommended. ${ }^{2,3,5,6,10,15}$

\section{Postoperative and rehabilitation protocols}

Post-operative treatment and follow-up has been only described by type IV studies. The most used scheme is shoulder immobilization with a sling for 2-6 weeks together with shoulder passive movements. ${ }^{5,8,10,17}$ At week 2 pendulum movements and passive motion is allowed. Active movements and sling removal is indicated at week $6^{5,15-17}$ Daily life activities are allowed progressively from week $6-12.15,17,24$ Full reintegration to endurance and sport activities is recommended only after 6 months. ${ }^{15,24}$

Becca Reisch published a detailed rehabilitation scheme in a FS, initiating rehabilitation therapy at week 6 after sling removal. He established a four stages goal based scheme: the protection stage (0-6 weeks), range of motion (6-10 weeks), dynamic strengthening (10-20 weeks) and daily living and sport returning $(+20$ weeks $) .{ }^{28}$

According to Tsung-li Lin observations of 39 FS patients, consolidation in the surgically fixed group was archived after 10 weeks. In the conservative treatment group or fracture consolidation was obtained after 12 weeks. ${ }^{18}$

Follow-up visits protocol varies, with a mean of 3 visits with control X-Rays in the first 12 weeks, (weeks: $1,2,6 .{ }^{8}$ or $2,6,12 .{ }^{17}$ ). Complete follow-up after 6 months and a year is recommended. ${ }^{15}$

\section{Conclusion}

Floating shoulder injuries can be easily misdiagnosed; its diagnosis requires an integral assessment and complete CT workup.

The treatment of floating shoulder injuries requires the assessment of each morphological injury individually. Fixing clavicular fractures and acromio-clavicular dislocations first can reduce the other SSSC injury. Displacement, medialization and the Glenopolar angle are the main criteria in decision making. A floating shoulder classification or treatment protocol is needed.

\section{Acknowledgements}

We thank Lilia Karime del Callejo Castillo Luna for her English style correction.

\section{Conflicts of interest}

All the Authors state they do not have any conflict of interest related to this article and have not receive any financial support.

\section{References}

1. Owens BD, Goss TP The floating shoulder. J Bone Jt Surg Br 2006;88(11):1419-1424.
2. Noort A Van, Werken C Van Der The floating shoulder. Injury. 2006;37:218-227.

3. Augusto C, García C, Villanueva JM et al. Tratamiento del hombro flotante: experiencia de ocho casos. Acta Ortopédica Mex. 2007;21(6):333-337.

4. Wright DEP, Johnstone AJ The Floating Shoulder Redefined. J Trauma. 2010;68(1):E26-E29.

5. Pailhes RG, Bonnevialle N, Laffosse J et al. Floating shoulders: Clinica and radiographic analysis at a mean follow-up of 11 years. Int J Shoulder Surg. 2013;7(2):59-64.

6. DeFranco MJ, Patterson BM The floating shoulder. J Am Acad Orthop Surg. 2014;14(8):499-509.

7. Butcher NE, Balogh ZJ Update on the definition of polytrauma. Eur $J$ Trauma Emerg Surg pp. 2014;107-111.

8. Friederichs J, Morgenstern M, Bühren V Scapula fractures in complex shoulder injuries and floating shoulders: a classification based on displacement and instability. J Trauma Manag Outcomes. 2014;8(16):4-9.

9. Toro JB, Helfet DL Surgical Management of the Floating Shoulder. Tech Shoulder Elb Surg. 2004;5(2):116-121.

10. Yadav V, Khare GN, Kumaraswamy V et al. A prospective study comparing conservative with operative treatment in patients with a "floating shoulder" including assessment of the prognostic value of the glenopolar angle. Bone Jt J. 2013;95:815-819.

11. Williams GR, Naranja J, Klimkiewicz J et al. The floating shoulder: a biomechanical basis for classification and management. J Bone Jt Surg Am. 2001;83(8):1182-1187.

12. Arksey H, Malley LO Scoping Studies: Towards A Methodological Framework. White Rose Research Online, p. 2005;19-32.

13. Levac D, Colquhoun H, O'Brien KK Scoping studies: advancing the methodology. Implement Sci. 2010;5:69.

14. Colquhoun HL, Levac D, O'Brien KK et al. Scoping reviews : time for clarity in definition, methods, and reporting. J Clin Epidemiol. 2014;67(12):1291-1294.

15. Márquez DP, Bernal C, López F Floating shoulder. Rev Andal Med Deport. 2014;7:132-135.

16. Elmadag M, Ceylan HH, Bilsel K et al. Floating Shoulder : Ipsilateral Clavicle, Scapular Body And Glenoid Fracture . A Case Report. Malaysian Orthop J. 2012;6(4):35-37.

17. Gilde AK, Hoffmann MF, Sietsema DL et al. Functional outcomes of operative fixation of clavicle fractures in patients with floating shoulder girdle injuries. J Orthop Traumatol. 2015;16(3):221-227.

18. Lin TL, Li YF, Hsu CJ et al. Clinical outcome and radiographic change of ipsilateral scapular neck and clavicular shaft fracture: comparison of operation and conservative treatment. J Orthop Surg Res. 2015;10(9):1-7.

19. Vogels J, Pommier N, Cursolle J et al. External fixator used to treat a floating shoulder injury caused by gunshot wound. Chir Main. 2014;33(5):370-374.

20. Izadpanah K, Jaeger M, Maier D et al. The floating shoulder-Clinical and radiological results after intramedullary stabilization of the clavicle in cases with minor displacement of the scapular neck fracture. $J$ Trauma. 2012;72(2):E8-E13.

21. Edwards SG, Whittle AP, Wood GW Nonoperative treatment of ipsilateral fractures of the scapula and clavicle. J Bone Joint Surg Am. 2000;82(6):774-780.

22. Kim K, Rhee K, Shin $\mathrm{H}$ et al. Can the Glenopolar Angle be Used to Predict Outcome and Treatment of the Floating Shoulder? J Trauma. 2008;64(1):174-178. 
23. Romero J, Schai P, Imhoff AB Scapular neck fracture - The influence of permanent malalignment of the glenoid neck clinical outcome. Arch Orthop Trauma Surg. 2001;121(6):313-316.

24. Lyons RP Open reduction and internal fixation of os acromion fractureseparation as a component of a floating shoulder injury : A case report. $J$ Shoulder Elb Surg. 2010;19(7):e18-e21.

25. Rodríguez IM, Mora J, Mari IT Triple rotura del complejo suspensorio del hombro. Rev Esp Cir Ortop Traumatol. 2016;57(5):371-374.
26. Goss TP Fractures of the glenoid neck. J Shoulder Elb Surg. 1994;3(1):42-52.

27. Izadpanah K, Jaeger M, Maier D et al. The floating shoulder-clinical and radiological results after intramedullary stabilization of the clavicle in cases with minor displacement of the scapular neck fracture. J Trauma Acute Care Surg. 2012;72(2):E8-E13.

28. Reisch B, Fischer J Rehabilitation of a patient with "floating shoulder" and associated fractures: A case report. J Trauma Acute Care Surg. 2012;28(7):542-551. 